\title{
Molecular Properties Obtained by Analysis of Electronic Spectra Containing Interference Dips. Comparisons of Analytical Equations and Exact Models Based on Coupled Potential Energy Surfaces
}

\author{
Guillaume Bussière and Christian Reber* \\ Département de chimie, Université de Montréal, Montréal, QC H3C 3J7 \\ Daniel Neuhauser,* Derek A. Walter, and Jeffrey I. Zink* \\ Department of Chemistry and Biochemistry, University of California, Los Angeles, California 90095
}

Received: August 8, 2002; In Final Form: October 8, 2002

\begin{abstract}
Interference dips in electronic absorption spectra caused by coupling between excited states are calculated and interpreted using three methods: an analytical expression, an integratable expression, and wave packet propagation. All of the analyses give similar results. The dips (distantly related to Fano's antiresonance) are caused by spin-orbit coupling between the states involved in spin-forbidden transitions with narrow bandwidths and the state involved in a spin-allowed transition with a large bandwidth caused by progressions in normal vibrational modes that are displaced between the excited and ground electronic states. The analytical expression involves the assumptions that only one vibrational eigenstate of the "forbidden" electronic state is involved and that the broad background is represented by a Lorentzian-type function. The integratable expression replaces the Lorentzian function by one that represents the band shape caused by a progression of unresolved vibronic peaks. The wave packet propagation method is exact for the model. Analytical expressions for multiple interfering states and integratable expressions for multiple electronic states and multiple vibrational modes are derived. The spectra of $\mathrm{Ni}\left(\mathrm{H}_{2} \mathrm{O}\right)_{6}{ }^{2+}$ and octahedral $\mathrm{CrO}_{6}{ }^{9-}$ units in $\mathrm{ZrO}_{2} \cdot 33 \% \mathrm{Y}_{2} \mathrm{O}_{3}$ doped with chromium(III) are analyzed. Physical meanings and mathematical origins of the interference dips are interpreted.
\end{abstract}

\section{Introduction}

Interference dips, ${ }^{1,2}$ sharp absorbance decreases in the electronic absorption spectra of metal-containing compounds and crystals at the energies where absorbances with very narrow bandwidths overlap a broad band, are frequently observed., . $^{1,21}$ These dips are very common in the spectra of octahedral chromium(III) complexes where the narrow absorption bands arise from intraconfigurational transitions and the broad band from $\pi^{*}$ to $\sigma^{*} \mathrm{~d}-\mathrm{d}$ transitions. The dips are found not only in the spectra of chromium-containing molecules but also in the spectra of crystals in which chromium is substituted for another ion in the lattice. ${ }^{4-6,8,10,11,14,16,19-21}$ Spectra of other $\mathrm{d}^{3}$ metal ions, especially those from vanadium(II), ${ }^{3,9}$ also exhibit dips; the state energies in ions with this electron configuration are frequently close to each other, and the bands overlap to produce the effect. Dips are not limited to $\mathrm{d}^{3}$ metal ions; examples are also found in the spectra of $\mathrm{d}^{8}$ nickel compounds, ${ }^{22} \mathrm{~d}^{2}$ chromium(IV) ions doped into a crystal lattice with tetrahedral sites, ${ }^{13}$ and $\mathrm{d}^{4}$ manganese(III) ions doped into oxide lattices. ${ }^{17,18}$ Dips are also common in the spectra of lanthanide compounds where sharp $\mathrm{f}-\mathrm{f}$ transitions overlap broad $\mathrm{d}-\mathrm{f}$ bands. Important examples are found in the spectra of europium(II) compounds and europium(II) doped in crystal lattices. ${ }^{7}$

Two examples of interference dips that are analyzed in this paper are shown in Figure 1. The decrease of the absorbance denoted by the arrows in the spectra is unexpected because the spectra arising from transitions to two or more electronic states are often the sum of the absorptions from transitions to each state and should increase the total absorbance. Dips in the intensities of broad absorption bands in atomic spectroscopy

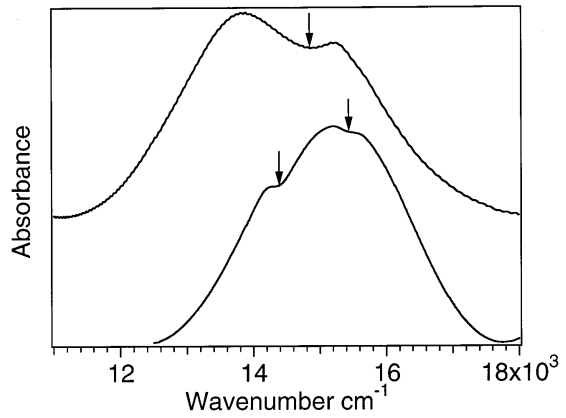

Figure 1. Absorption spectra illustrating interference dips. Top: Absorption spectrum of $\mathrm{Ni}\left(\mathrm{H}_{2} \mathrm{O}\right)_{6}{ }^{2+}$ at room temperature showing the region of the ${ }^{3} T_{1}$ band. The dip (indicated by the arrow) at 14850 $\mathrm{cm}^{-1}$ is caused by interference with the ${ }^{1} \mathrm{E}$ state. Bottom: Absorption spectrum of octahedral $\mathrm{CrO}_{6}{ }^{9-}$ units in $\mathrm{ZrO}_{2} \cdot 33 \% \mathrm{Y}_{2} \mathrm{O}_{3}$ doped with chromium(III) at $5 \mathrm{~K}$ in the region of the ${ }^{4} \mathrm{~T}_{2}$ band. The two dips (indicated by the arrows) are caused by interference with the ${ }^{2} \mathrm{E}$ state at $14344 \mathrm{~cm}^{-1}$ and the ${ }^{2} \mathrm{~T}_{1}$ state at $15430 \mathrm{~cm}^{-1}$.

were interpreted by Fano et al. and are known as "Fano antiresonances". ${ }^{23,24}$ Until recently, the interference dips in the spectra of molecules were interpreted by applying equations developed for atomic spectroscopy ${ }^{23,24}$ even though the breadth of the absorption bands of the former are caused by unresolved progressions in vibronic bands, whereas those in the latter are due to the ionization continuum.

The first analyses of interference dips in molecular spectra based on molecular properties were reported recently.,21,25 The spectra were treated in terms of spin-orbit coupled ligand field excited states. Potential surfaces for these states were constructed 


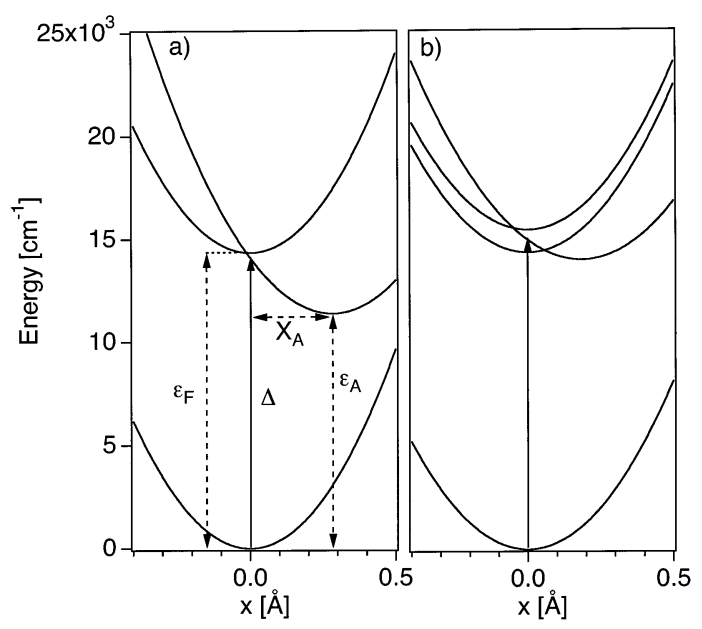

Figure 2. Potential surfaces for the ground and excited states producing the interference dips. (a) Potential surfaces for $\mathrm{Ni}\left(\mathrm{H}_{2} \mathrm{O}\right)_{6}{ }^{2+}$. The arrow illustrates the transition from the ground state to the displaced ${ }^{3} \mathrm{~T}_{1}$ state. A transition to the undisplaced ${ }^{1} \mathrm{E}$ state is spin forbidden. Quantities used to calculate spectra with the numerical values in Table 1 are given. (b) Potential surfaces for octahedral $\mathrm{CrO}_{6}{ }^{9-}$ units in $\mathrm{ZrO}_{2} \cdot 33 \% \mathrm{Y}_{2} \mathrm{O}_{3}$ doped with chromium(III). The arrow illustrates the transition from the ground state to the displaced ${ }^{4} \mathrm{~T}_{2 \mathrm{~g}}$ state. Transitions to the undisplaced ${ }^{2} \mathrm{E}$ and ${ }^{2} \mathrm{~T}_{1}$ states are spin forbidden.

from known vibrational and electronic properties of the molecules, and the spectra were calculated by time-dependent theory and wave packet propagation on the coupled surfaces. ${ }^{1,12,25-28}$ Examples of the potential surfaces used to analyze the spectra in this paper are shown in Figure 2. These wave packet propagation calculations, although exact in the framework of the model, are not as simple or convenient to carry out as those based on an analytic expression.

A simple analytical expression for the intensity decrease in molecular absorption spectroscopy was recently derived..$^{2}$ It uses the assumption that not all vibrational states are populated. Specifically, it assumes that the electronic state with its potential surface minimum at the same position in normal coordinate space as the ground state does not couple directly to the ground state, but only indirectly through its coupling to the final state of the spin-allowed d-d transition. In this "dark" state, the highenergy vibrational states are not considered important, and only the ground vibrational state is used. This simplification, which was numerically verified, not only provides an equation that can be used to calculate the spectrum on a graphing calculator but also provides an analytical "line shape function", i.e., the difference spectrum between that of the coupled system and the uncoupled spectrum with no interference dip. The latter was cast into an ostensibly equal form to Fano's line shape equation, but the physical meaning of the parameters is different. ${ }^{2}$

In many experimental examples, more than two states are coupled and more than one dip is observed in the spectrum. This situation is often found in the spectra of chromium(III) complexes and crystals. An example is shown in Figure 1. Exact calculations based on wave packet propagation for three or more electronic states are time-consuming and require significant computer power and careful evaluation of the numerical results to avoid artifacts from errors accumulating at each time step. Simple methods and analytical expressions are needed.

In this paper, we report the results of analyses of interference dips in the spectra of $\mathrm{Ni}\left(\mathrm{H}_{2} \mathrm{O}\right)_{6}{ }^{2+}$ and octahedral $\mathrm{CrO}_{6}{ }^{9-}$ units in $\mathrm{ZrO}_{2} \cdot 33 \% \mathrm{Y}_{2} \mathrm{O}_{3}$ doped with chromium(III) ${ }^{20}$ shown in Figure 1. The absorption spectrum of the former contains one pronounced dip, whereas that of the latter contains two. The nickel spectrum is fit and interpreted by using the analytical expression and the exact wave packet propagation calculation. The former reveals that the Lorentzian function used in the analytical expression provides an accurate and quantitative analysis of the molecular properties producing the dips but does not accurately fit the wings of the spectrum. To better fit the entire spectrum, an integratable expression is derived that is based on a band shape dominated by vibronic progressions. The molecular properties obtained from all three calculations are compared and discussed. In the case of the chromium(III) spectrum, new analytical and integratable equations are derived to treat correctly the case of three or more interacting states. The molecular properties obtained from these calculations are compared and discussed, and the errors inherent in assuming that two or more dips are independent of each other are highlighted. Finally, the physical and mathematical meanings and origins of the interference dips are interpreted. The dips are a form of destructive quantum interference.

\section{Theory}

The theory is based on the coupling between a forbidden state and an allowed state where the forbidden state's potential surface is undisplaced from that of the ground state and the allowed state's potential surface is highly displaced. In the case of spectra of many metal-containing compounds, the forbidden surface is the final state of an intraconfigurational "spin flip" transition where the spin multiplicity changes but no change in orbital occupancy is involved. The allowed surface frequently corresponds to the $\mathrm{t}_{2 \mathrm{~g}}$ to $\mathrm{e}_{\mathrm{g}} \pi^{*}$ to $\sigma^{*}$ antibonding transition. This type of transition significantly weakens the metal-ligand bonding resulting in a broad absorption band. A schematic of the diabatic potential surfaces that illustrate the model and illustrations of the most important parameters are shown in Figure 2.

The total electron-nuclear Hamiltonian for the coupled excited states is

$$
H=\frac{p^{2}}{2 M}\left[\begin{array}{ll}
1 & 0 \\
0 & 1
\end{array}\right]+\left[\begin{array}{ll}
\frac{1}{2} M \omega_{\mathrm{F}}{ }^{2} x^{2}+\epsilon_{\mathrm{F}} & \gamma \\
\gamma & \frac{1}{2} M \omega_{0}{ }^{2}\left(x-x_{\mathrm{A}}\right)^{2}+\epsilon_{\mathrm{A}}
\end{array}\right]
$$

where $\mathrm{M}$ is the oscillator's mass, $\omega_{0}$ and $\omega_{\mathrm{F}}$ are the frequencies on the allowed and forbidden states, $\mathrm{x}$ is the vibrational coordinate, $\mathrm{x}_{\mathrm{A}}$ is the shift of the minimum of the excited state's minimum upon excitation, $\epsilon_{\mathrm{F}}$ and $\epsilon_{\mathrm{A}}$ are the forbidden and allowed states' minimum potential energy, and $\gamma$ is the spinorbit coupling between the two states (assumed to be coordinate independent).

The absorption cross section $\sigma(\omega)$ as a function of frequency is given by

$$
\begin{aligned}
\sigma(\omega) & =\frac{1}{2 \pi} \int_{-\infty}^{\infty}\left\langle\Psi_{0}\left|\mathrm{e}^{-i(H-\omega) t-\Gamma|t|}\right| \Psi_{0}\right\rangle \mathrm{d} t \\
& =\frac{1}{\pi} \operatorname{Im}\left\langle\Psi_{0}\left|(H-\omega-i \Gamma)^{-1}\right| \Psi_{0}\right\rangle
\end{aligned}
$$

where $\Gamma$ is a phenomenological damping factor that represents the effects of coupling to other molecules or to the solvent, $\Psi_{0}$ $=[0,1]^{\mathrm{T}} \psi_{0}$ is the initial wave function that is localized on the allowed electronic state $\mathrm{A}$, and $\psi_{0}$ is the ground vibrational state of the ground electronic state. 
A. Approximate Analytical Equation. An analytical expression for calculating interference dips has recently been derived. ${ }^{2}$ With the assumption that only a single vibrational state from the forbidden electronic state is involved, the absorption cross section is

$$
\sigma(\omega)=-\frac{1}{\pi} \operatorname{Im}\left(\frac{\beta}{1-\gamma^{2} \alpha \beta}\right)
$$

where $\beta$ is the cross section of the broad spin-allowed transition, $\alpha$ is the cross section of the narrow spin-forbidden transition and $\gamma$ is the coupling (spin-orbit coupling) between the two. The equations for $\alpha$ and $\beta$ are

$$
\begin{gathered}
\alpha=\frac{1}{\omega-\epsilon_{\mathrm{F}}+i \Gamma} \\
\beta=\frac{1}{\omega-\Delta+i \sqrt{\omega_{0} \lambda}}
\end{gathered}
$$

where $\Delta$ is the maximum of the broad absorption transition to the displaced excited-state potential surface and $\lambda$ is the energy difference between $\Delta$ and the lowest-energy level $\epsilon_{\mathrm{A}}$ of the displaced excited state, as illustrated in Figure $2 \mathrm{a} . \Gamma$ is the damping factor that determines the width of individual vibronic lines. The difference spectrum is given by

$$
\sigma_{\text {diff }}(\omega)=\sigma(\omega)-\sigma_{0}(\omega)=\frac{\gamma^{2}}{\pi} \operatorname{Im} \frac{\alpha \beta^{2}}{1-\gamma^{2} \beta \alpha}
$$

This spectrum is the difference between the spectrum with and without coupling and gives the "line shape function" that shows how the spectrum arising from the transition to the allowed state is changed by the interference from the forbidden state. It consists of an interference dip bounded by two peaks. The physical meaning of the interference is discussed in the final section of this paper.

The analytical expression for calculating the spectrum when two undisplaced forbidden states are present has not been presented earlier. It is derived in Appendix A and is

$$
\sigma(\omega)=-\frac{1}{\pi} \operatorname{Im}\left(\frac{\beta}{1-\left(\gamma_{1}^{2} \alpha_{1}+\gamma_{2}^{2} \alpha_{2}\right) \beta}\right)
$$

where $\gamma_{1}$ and $\gamma_{2}$ are the coupling constants of forbidden state $\alpha_{1}$ and forbidden state $\alpha_{2}$ with the allowed state. Note that the effect of two forbidden states cannot be calculated separately. The application of this equation to the interference dips in the spectrum of $\mathrm{a} \mathrm{d}^{3}$ chromium(III) complex is discussed in Section IV.

B. Integratable Equation. The simple analytical expressions discussed above assume that the underlying background spectrum can be approximated by a Lorentzian function. For spectra such as those shown in Figure 1, corresponding to the typical resolution observed for transition metal compounds in solids or solution, this assumption is good for the intense part of the spectrum near the peak maximum, but it fails in the region of the weak absorbance "wings" of the spectrum. For highly resolved spectra, the approximation is inadequate.

The band shape of many transition metal compounds is caused by unresolved progressions of vibronic bands and is accurately represented by a smeared out Poisson distribution. An analytical equation has not been derived using a Poisson function. However, an integratable equation that represents exactly the background spectrum caused by displaced harmonic potential surfaces can be derived (see Appendix B.) The final equation for $\beta$ is

$$
\begin{gathered}
-i \beta(\omega)=\int_{0}^{\infty} \exp \left(-\sum_{j} \zeta_{i}\left(1-\exp \left(-i \omega_{j} t\right)\right)+\right. \\
\left.i t\left(\omega-\frac{1}{2} \sum_{j} \omega_{j}\right)-\Gamma t\right) \mathrm{d} t \\
\zeta_{j} \equiv \frac{\left(x_{A j}-x_{D j}\right)^{2}}{2 \sigma j^{2}}
\end{gathered}
$$

where $\sigma_{j}$ is the width at half-height. This equation is more accurate than the analytic equations above, but still suffers from the problem that the tail of the spectrum falls off slowly because of the Lorentzian line-shape. One approach to solve that problem is to change the fall off from exponential $\left(\mathrm{e}^{-\Gamma t}\right)$ to Gaussian in the integrand for eq 1.7. This would not be completely consistent, because the derivation of eqs 1.3 and 1.6 relies on the expansion of the Green's function $1 /(E-H+i \Gamma)$, associated with an exponential falloff.

C. Exact Calculations. The Hamiltonian can be explicitly evaluated and the spectra calculated by using time-dependent techniques. ${ }^{1,26}$ Because no approximation needs to be made, such calculations are exact within the framework of the model. One of the goals of this work is to compare the molecular parameters that are obtained by fitting spectra having interference dips with the simple analytical expression, the integratable equation, and the exact calculation. The simple equation, implemented on a graphing calculator, yields reasonable values of the molecular properties.

\section{Analysis of the Interference Dip in The Spectrum of $\mathrm{Ni}\left(\mathrm{H}_{2} \mathrm{O}\right)_{6}{ }^{2+}$}

The spectrum of $\mathrm{Ni}\left(\mathrm{H}_{2} \mathrm{O}\right)_{6}{ }^{2+}$ shown in Figure 1 contains a striking example of an interference dip, the minimum observed at $14850 \mathrm{~cm}^{-1}$. Two peaks are observed on both sides of the dip at 13900 and $15400 \mathrm{~cm}^{-1}$. The total width at half-height of the double-peaked band is about $3100 \mathrm{~cm}^{-1}$.

The interpretation of this spectrum has had a long and controversial history. ${ }^{22,29-33}$ Originally the two peaks in the spectrum were interpreted as resulting from transitions to the ${ }^{3} \mathrm{~T}_{1}\left({ }^{3} \mathrm{~F}\right)$ and ${ }^{1} \mathrm{E}$ excited states. However, the intraconfigurational transition to the ${ }^{1} \mathrm{E}$ state is expected to be much narrower than either of the peaks in the spectrum. Recently, a low temperature spectrum revealed highly resolved vibronic structure and was analyzed in detail in terms of two coupled states. ${ }^{22}$ The focus in this section will be on the destructive interference that leads to the dip that separates the two peaks.

A. Analysis Using the Analytical Expression. The calculated spectrum using eq 1.3, the analytical expression, is shown in Figure 3a. The starting point for fitting the experimental spectrum requires the quantification of the potential surfaces in Figure 2a. The allowed surface determines the position and the width of the underlying background spectrum given by $\beta$. The energy of the peak maximum is approximately at the center of the spectrum, in this case at about $14300 \mathrm{~cm}^{-1}$. The forbidden surface determines the position of the dip; a simple starting point is to position the energy $\epsilon_{\mathrm{F}}$ at the minimum of the dip, in this case at about $14800 \mathrm{~cm}^{-1}$. The coupling $\gamma$ is the spin-orbit coupling. For the $\mathrm{E}\left({ }^{1} \mathrm{E}\right)$ and $\mathrm{E}\left({ }^{3} \mathrm{~T}_{2 \mathrm{~g}}\right)$ states of $\mathrm{Ni}(\mathrm{II})$, the coupling is $660 \mathrm{~cm}^{-1}$. The damping factor $\Gamma$ is treated as a fitting parameter; for transition metal complexes in solution, it typically will have values between 100 and $1000 \mathrm{~cm}^{-1}$. 

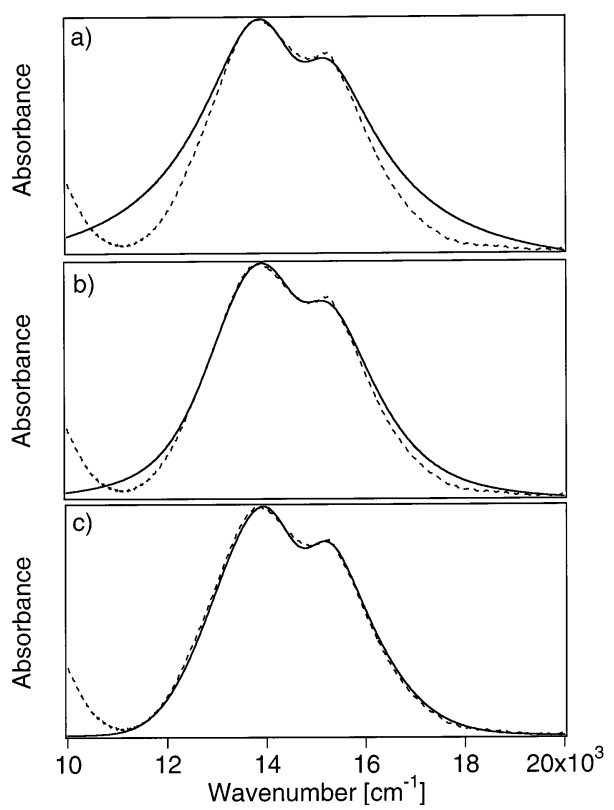

Figure 3. Calculated (solid lines) and experimental (dashed lines) spectra of $\mathrm{Ni}\left(\mathrm{H}_{2} \mathrm{O}\right)_{6}{ }^{2+}$ using the parameters given in Table 1. (a) Spectrum calculated using the analytical equation. (b) Spectrum calculated using the integratable equation. (c) Spectrum calculated by wave packet propagation on the surfaces shown in Figure 2.

TABLE 1: Parameters Used to Calculate the Spectrum of $\mathrm{Ni}\left(\mathrm{H}_{2} \mathrm{O}\right)_{6}{ }^{2+}$ in Figure 3

\begin{tabular}{lccc}
\hline parameter & $\begin{array}{c}\text { analytical } \\
\text { equation }\end{array}$ & $\begin{array}{c}\text { integratable } \\
\text { equation }\end{array}$ & $\begin{array}{c}\text { wave packet } \\
\text { calculation }\end{array}$ \\
\hline$\epsilon_{\mathrm{A}}, \mathrm{cm}^{-1}$ & & 11375 & 10400 \\
$\Delta, \mathrm{cm}^{-1}$ & 14325 & 14341 & 14333 \\
$\epsilon_{\mathrm{F}}, \mathrm{cm}^{-1}$ & 14757 & 14780 & 14650 \\
$\lambda, \mathrm{cm}^{-1}$ & 7620 & & \\
$\mathrm{X}_{\mathrm{A}} \AA^{-1}$ & & 0.283 & 0.313 \\
$\Gamma, \mathrm{cm}^{-1}$ & 630 & 615 & 300 \\
$\omega_{\mathrm{F}}, \mathrm{cm}^{-1}$ & 380 & 380 & 380 \\
$\omega_{\mathrm{A}}, \mathrm{cm}^{-1}$ & 380 & 357 & 380 \\
$\gamma, \mathrm{cm}^{-1}$ & 550 & 420 & 380
\end{tabular}

The spectrum is calculated by using the initial trial values of the parameters and comparing the resulting calculated spectrum to the experimental spectrum. The parameters are varied until a best fit is achieved. The best fit spectrum (solid line) is compared to the experimental spectrum (dashed line) in Figure $3 \mathrm{a}$. For the fit shown, the value of the vibrational frequency is fixed at $380 \mathrm{~cm}^{-1}$ and the value of the spin-orbit coupling and the damping are varied independently to achieve the best leastsquares fit. The parameter values for the best fits are shown in Table 1.

The fit demonstrates quantitatively that the interference is a result of spin-orbit coupling (with a value within the range determined by independent experiments), state energies consistent with known crystal field energies, and the known vibrational frequency of the totally symmetric $\mathrm{Ni}-\mathrm{O}$ normal mode. The calculated damping factor of $630 \mathrm{~cm}^{-1}$ is well within the range of damping factors for metal compounds in solution. The fit demonstrates that the interference is interpretable in terms of physically meaningful molecular parameters.

The width of the antiresonance dip is sensitive to the damping factor $\Gamma$ and increases as $\Gamma$ increases. A change of $\pm 100 \mathrm{~cm}^{-1}$ causes a poorer fit. The depth of the dip is very sensitive to the magnitude of the coupling constant $\gamma$ and increases as $\gamma$ increases. A change of $\pm 10 \mathrm{~cm}^{-1}$ causes a poorer fit. When the forbidden state is near the maximum of the allowed band, a small $\Gamma$ with a large coupling constant leads to a sharp, deep and well-defined antiresonance. If a large $\Gamma$ and a small coupling constant are used, the antiresonance dip diminishes and the resulting band shape becomes very similar to a featureless Lorentzian.

The goodness of the fit is primarily determined by the interplay between these two parameters. (The relative energies of the two states are also important and determine the position of the dip, but once these energies are determined the fitting becomes dependent on the coupling and damping.) In the fit of the nickel spectrum, one unique set of parameters produces the best least-squares fit. Varying the spin-orbit coupling constant by $5 \%$ and the damping factor by $20 \%$ significantly decreased the agreement between fit and experiment.

The criterion for the best fit was weighted toward the upper portion of the spectrum to emphasize the region around the interference dip and its surrounding peaks. The analytical equation uses a Lorentzian function for the spectrum of the allowed state. This function does not properly model the bandwidth of the absorption spectrum shown in Figure 1; the "wings" of the function are broader at low and high frequencies than the shape caused by the displaced harmonic oscillator. Using this function does produce reliable values of the parameters governing the interference as will be discussed below. It is important to recognize that the experimental spectrum contains contributions from other spin-orbit states in addition to the states of E symmetry from both the ${ }^{3} \mathrm{~T}_{1}$ and ${ }^{1} \mathrm{E}$ states that produce the interference dip in the spectrum of $\mathrm{Ni}\left(\mathrm{H}_{2} \mathrm{O}\right)_{6}{ }^{2+}$. The other spin-orbit states do not couple to the forbidden state and thus do not contribute to the dip, but they do contribute to the total absorption bandwidth. In the twostate model discussed above, these contributions are not explicitly included. Thus, the calculated displacement is an upper limit; a slightly smaller value would result if small contributions from the other uncoupled spin-orbit states were added to the calculated spectrum. The neglect of these contributions is estimated to cause a $0.03 \AA$ error in the value of the displacement $\mathrm{X}_{\mathrm{A}}$. The value of $\mathrm{X}_{\mathrm{A}}$ from the analysis of the single-crystal spectrum is $0.30 \AA .22$

B. Analysis Using the Integratable Equation. An analytical equation has not been derived for the case of a band shape caused by unresolved progressions of vibronic bands, but an integratable equation that represents the background spectrum caused by displaced harmonic potential surfaces is derived in Appendix B and given in eq 1.7. For the octahedral complexes discussed in this paper, only one normal coordinate is used to calculate the band shape. However, eq 1.7 can be used for multiple normal coordinates when spectra of lower symmetry metal complexes are analyzed.

The best fit to the absorption spectrum of the nickel complex is shown in Figure $3 b$, and the values of the parameters are given in Table 1. The values are very similar to those obtained by fitting the spectrum with the analytical equation. Detailed comparisons are discussed in section D below.

C. Analysis Using Wave Packet Propagation. The spectrum resulting from the two-state model can be calculated exactly by using time-dependent wave packet propagation techniques. The methods and insight obtained from these procedures were discussed extensively. 1,12,25,27,28 The best fit obtained by using the exact calculation is shown in Figure $3 \mathrm{c}$ and the values of the parameters are given in Table 1.

D. Comparisons of the Calculations. The molecular properties that are deduced by fitting the experimental spectra using the three different methods are very similar as shown in Table 1. The analytical expression, the simplest and fastest method 
to use, provides values that are the same within the experimental uncertainty (with the exception of the coupling constant) as those from the exact wave packet propagation fit. Most of the properties are constrained by the experimental data and are not freely variable fitting parameters.

The first step in fitting the experimental spectrum is to position the potential surfaces such that the energies of the allowed and forbidden states match those in the experimental spectrum. The energy of the forbidden intraconfigurational state is constrained by the position of the dip, and the energy of the allowed state is constrained by the broad overall spectrum. These values can be "tweaked" slightly (by less than $200 \mathrm{~cm}^{-1}$ out of $10000 \mathrm{~cm}^{-1}$ ) during the fitting procedure, but the values are highly constrained by the experimental data. In the three fits, the values of the energy of the forbidden state that gave the best fits to the position of the dip are within $130 \mathrm{~cm}^{-1}$ of each other as shown in Table 1. The energies of the peak maxima of the allowed state (defined by the position of the absorption band maximum calculated with no coupling) are within only $16 \mathrm{~cm}^{-1}$ of each other.

Another step in the fitting process is to fit the overall width of the spectrum. In the case of the analytical expression, the width is defined by the value of $\lambda$, whereas in the cases of the integratable equation and the exact wave packet propagation calculation, it is defined by both the vibrational frequency and the displacement of the allowed potential surface. In all cases, the final bandwidths are the same. In the latter two calculations, the displacements are within $0.03 \AA$ of each other. Note that the width depends on the vibrational frequency; in the calculations with the analytical equation and in the exact wave packet calculation, the vibrational frequencies for all states are fixed at $380 \mathrm{~cm}^{-1}$ as measured for the totally symmetric $\mathrm{Ni}-\mathrm{H}_{2} \mathrm{O}$ stretching mode by vibrational spectroscopy. This assumption is not necessary in the integratable and wave packet calculations, but it does not significantly affect the values of the parameters. We show this for the fit based on the integratable equation using the experimental vibrational energy for the ${ }^{3} \mathrm{~T}_{1}$ state determined from low-temperature single-crystal spectra ${ }^{22}$ and the value determined from Raman spectra for the ground and ${ }^{1} \mathrm{E}$ excited states.

The final steps in fitting the spectra involve varying the coupling and the damping to best fit the depth of the dip. The value of the damping is strongly constrained by the resolution in the spectrum. In the spectra of most transition metal complexes, the overall absorption bandwidth is determined primarily by the underlying vibronic structure (i.e., by the values of the vibrational frequency and the displacement), so the value of the damping is required to be large enough to wash out the vibronic structure but not large enough to contribute significantly to the overall bandwidth. In the calculations reported here, the damping $\Gamma$ contributes only a few hundred wavenumbers to the bandwidth. The damping is treated as a phenomenological factor that represents dephasing and loss of the wave packet to other vibrational modes and to the bath. The damping is not directly comparable between the exact wave packet calculation and the other two fitting methods, but the values determined by the fit given in Table 1 show that their contributions to the spectra are comparable.

The best fit values of the coupling are within twenty percent of each other. The magnitude, about $450 \mathrm{~cm}^{-1}$, is within the range of values that have been calculated for nickel(II) complexes.

E. Difference Spectra and the "Line Shape Function". A difference spectrum, a plot of the difference between the actual

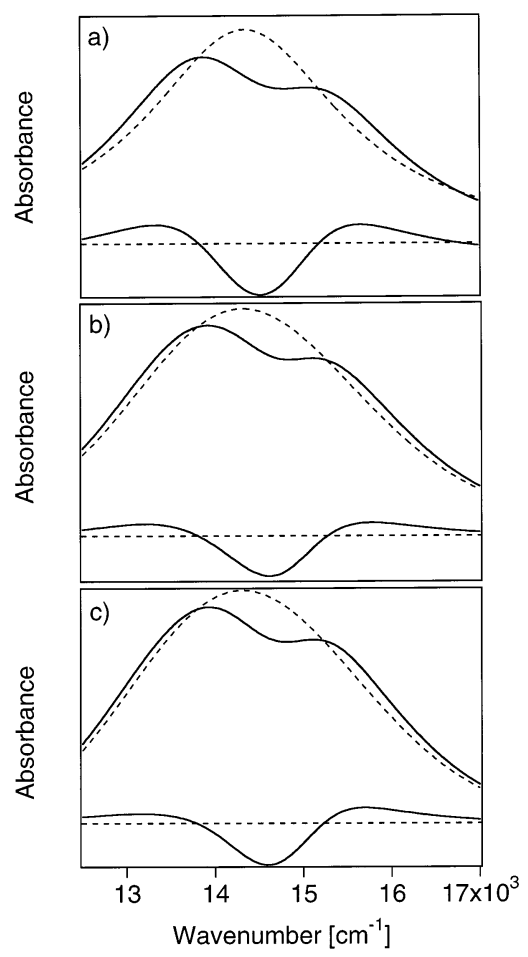

Figure 4. Difference spectra or "line-shape functions". In each panel, the top two spectra are calculated for $\mathrm{Ni}\left(\mathrm{H}_{2} \mathrm{O}\right)_{6}{ }^{2+}$ with and without spin-orbit coupling. The difference between the spectra is shown at the bottom of the panel. Note the "up-down-up" or "two peaks separated by a dip" shape of the difference spectrum. (a) Difference spectrum for the fit with the analytical equation. (b) Difference spectrum resulting from the fit using the integratable equation. (c) Difference spectrum obtained from the exact wave packet calculation.

spectrum including the interference dip and the assumed or calculated spectrum with no coupling and thus no dip, is often called the "line shape function". Difference spectra are a convenient way of illustrating the changes caused by the coupling. Difference spectra for $\mathrm{Ni}\left(\mathrm{H}_{2} \mathrm{O}\right)_{6}{ }^{2+}$ are shown in Figure 4 where a spectrum with nonzero coupling, a spectrum calculated with zero coupling, and the difference between them are plotted. The difference spectra always have the same pattern: the difference intensity is negative at the position of the dip and is positive at lower and higher energies. The difference spectrum can be simply described as two peaks surrounding a dip. ${ }^{2}$

It is tempting to try to fit a line shape function rather than the actual spectrum. The problem that arises is that the line shape function representing the "background" spectrum without coupling must be known in order to subtract it from the actual spectrum. A large number of assumptions must be made about the underlying background spectrum (the quantity $\beta$ ) including assumptions about its intensity, its width and the energy of its peak maximum. The difficulties are illustrated by the width of the "background" spectrum in Figure 4a, which is $2700 \mathrm{~cm}^{-1}$, significantly less than the width of $3100 \mathrm{~cm}^{-1}$ for the experimental band. This significantly narrower width cannot be accurately obtained from an intuitive choice of baseline or from an empirical fit. Errors or biases in choosing the background can lead to errors or misinterpretations of the shape function.

A common choice for the shape function is the "Fano line shape function", i.e., the difference spectrum derived by Fano for dips in atomic spectra. The traditional "Fano" function has either an "up-down" (constructive-destructive) or "down-up" shape as scanned from low to high energy. Artificially moving and scaling the background envelope can produce a "Fano" line 
shape. The correct difference spectrum in molecular spectroscopy must have an "up-down-up" shape as presented later. Most published interpretations of interference dips in molecular spectra assume Fano's equations are applicable, that the downup shape function is appropriate, and artificially choose a background that produces the desired shape. It is obvious that parameters obtained with this approach carry little significance because the equations are inappropriate and the choice of background is arbitrary. The difficulties of obtaining physical insight from this approach have been illustrated recently. ${ }^{34}$ The best fitting procedure fits the entire experimental spectrum, not a biased shape function.

\section{Analysis of the Interference Dips in the Spectrum of Chromium(III)}

The most common examples of interference dips in the absorption spectra of large molecules are found in the spectra of first row octahedral transition metal $\mathrm{d}^{3}$ complexes. The energies of the ${ }^{2} \mathrm{~T}_{1}$ and ${ }^{2} \mathrm{E}$ excited states are about the same as that of the ${ }^{4} \mathrm{~T}_{2}$ excited state. The former two states arise from intraconfigurational transitions within the $t_{2 g}$ orbitals, whereas the latter involves the $t_{2 g}$ to $e_{g}$ transition. The ${ }^{2} \mathrm{~T}_{1}$ and ${ }^{2} \mathrm{E}$ states cause multiple interference dips in the broad, spin-allowed band in the spectra of a large number of chromium(III) complexes and were first identified as interference effects (often labeled as Fano antiresonances) in the spectra of vanadium(II) ions doped into $\mathrm{KMgF}_{3}{ }^{3}$

The analytical expression needed to calculate the spectra containing two interference dips is derived in Appendix A. The equation (eq 1.6) is similar to that used for one interfering state, but the presence of the sum in the denominator shows that it is not simply the sum of successive applications of the equation for a single interfering state. Thus, spectra containing multiple interference dips cannot be calculated correctly by using sequentially the two-state equation that was appropriate for the nickel complex. It is computationally very time-consuming to carry out exact wave packet propagation calculations involving three coupled surfaces and multiple vibrational dimensions.

A. Fit with the Analytical Formula for Three Coupled States. The fitting procedure is similar to that used for two coupled states. The appropriate potential surfaces are shown in Figure $2 \mathrm{~b}$ and are quantified in the same manner as those used in the fit of the nickel complex's spectrum. For chromium(III), the energies of two forbidden and undisplaced surfaces are needed to determine the positions of the two dips; a meaningful starting point is to position the energies $\epsilon_{\mathrm{F}}$ at the minima of the dips, in this case at about 14300 and $15200 \mathrm{~cm}^{-1}$. The fitting is slightly more complicated because varying the parameters to fit one of the dips influences those needed to fit the other. The calculated and experimental spectra are compared in Figure 5a and the best fit values are given in Table 2 .

B. Analysis Using the Integratable Equations. An integratable equation (eq 1.7) that calculates the background band shape caused by a progression of vibronic bands from a displaced potential surface and the interference with two undisplaced potential surfaces (Figure 2b) is derived in Appendix $\mathrm{B}$. This equation more accurately calculates the absorbance in the "wings" of the spectrum as is the case in the twostate model. The fitting procedure using the equation parallels that for the nickel complex. Again, the fitting is slightly more complicated than it was for two states because varying the parameters to fit one of the dips influences those needed to fit the other. The calculated and experimental spectra are compared in Figure $5 \mathrm{~b}$ and the best fit values are given in Table 2.
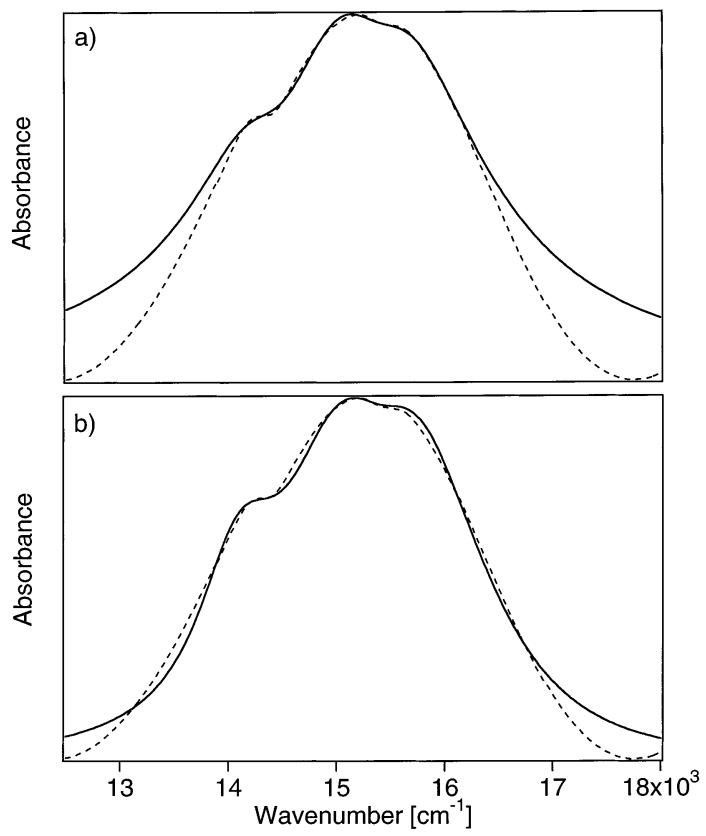

Figure 5. Calculated (solid lines) and experimental (dashed lines) spectra of octahedral $\mathrm{CrO}_{6}{ }^{9-}$ units in $\mathrm{ZrO}_{2} \cdot 33 \% \mathrm{Y}_{2} \mathrm{O}_{3}$ doped with chromium(III) using the parameters given in Table 2. (a) Spectrum calculated using the analytical equation. (b) Spectrum calculated using the integratable equation.

TABLE 2: Parameters Used to Calculate the Absorption Spectrum of $\mathrm{ZrO}_{2} \cdot 33 \% \mathrm{Y}_{2} \mathrm{O}_{3}$ Doped with $2 \% \mathrm{Cr}^{3+}$ in Figure 5

\begin{tabular}{lrc}
\hline \multicolumn{1}{c}{ parameter } & $\begin{array}{c}\text { analytical } \\
\text { equation }\end{array}$ & $\begin{array}{c}\text { integratable } \\
\text { equation }\end{array}$ \\
\hline$\epsilon_{\mathrm{A}}, \mathrm{cm}^{-1}$ & & 13980 \\
$\Delta, \mathrm{cm}^{-1}$ & 15166 & 15101 \\
$\epsilon_{\mathrm{F} 1}, \mathrm{~cm}^{-1}$ & 14379 & 14330 \\
$\epsilon_{\mathrm{F} 2}, \mathrm{~cm}^{-1}$ & 15406 & 15430 \\
$\lambda, \mathrm{cm}^{-1}$ & 3620 & \\
$\mathrm{X}_{\mathrm{A}}, \mathrm{A}^{-1}$ & & \\
$\Gamma, \mathrm{cm}^{-1}$ (for states 1 and 2) & 420 & 0.184 \\
$\omega_{\mathrm{F}}, \mathrm{cm}^{-1}$ & 470 \\
$\omega_{\mathrm{A}}, \mathrm{cm}^{-1}$ & 370 & 370 \\
$\gamma_{1}, \mathrm{~cm}^{-1}$ (state 1$)$ & 370 & 345 \\
$\gamma_{2}, \mathrm{~cm}^{-1}$ (state 2) & 325 & 395 \\
& 241 & 245
\end{tabular}

C. Comparisons of the Calculations. The values of the fitting parameters that are used to fit the spectrum using the two different methods are very similar as shown in Table 2. Most of the discussion about the fitting procedures and the constraints on the parameters in the section on the nickel complex also applies to the chromium complex. In the case of the chromium complex, the values of the energies of the forbidden states that gave the best fits to the positions of the dips are within less than $50 \mathrm{~cm}^{-1}$ of each other. The energies of the peak maxima of the allowed state are within less than 65 $\mathrm{cm}^{-1}$ of each other. The achievement of the best fit to the spectra containing two dips required more effort than that for the spectrum containing one dip, probably because changing one of the parameters for a given state affected more than one other state.

D. Comparison of Two Successive Two-State Fits with the Three-State Fit. The presence of two or more interference dips is common in the spectra of chromium(III) complexes. The usual fitting procedure found in the literature treats the spectrum one dip at a time. This type of successive fitting of each dip is not correct as can be seen from the analytical expression given by eq 1.6. This equation is not the sum of two eqs 1.3 and thus the three-state spectrum is not the sum of two two-state spectra. 

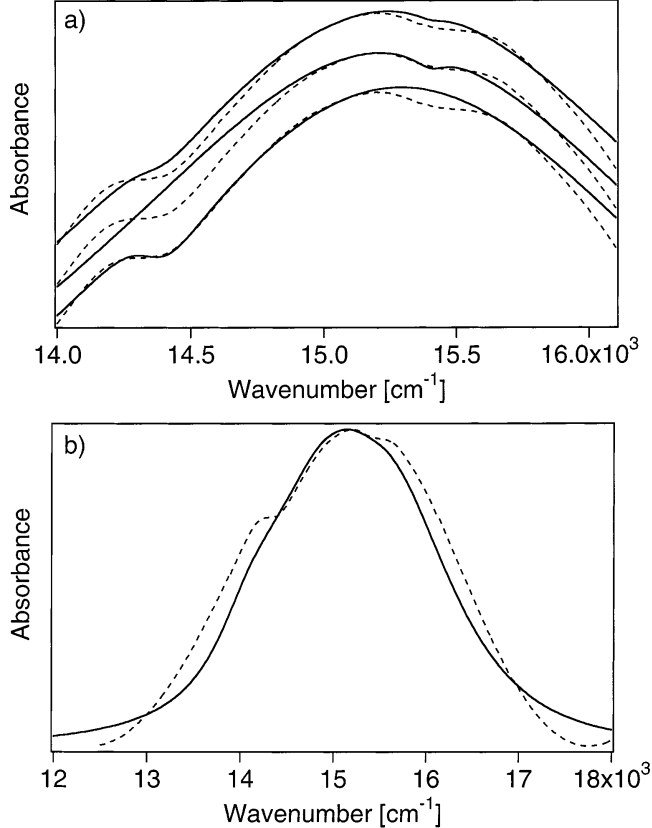

Figure 6. Calculated spectra using two sequential applications of the equations for one dip. (a) The three pairs of traces show the best fit to the lowest energy interference dip (bottom), the highest energy interference dip (middle), and the sum of the two traces normalized to the same area as the experimental spectrum (top). In all cases, the experimental spectrum is shown by the dashed lines. Note that the sum is not a good fit and that the depths of the dips are poorly reproduced. (b) The solid line is the spectrum calculated by using the equation for one dip successively with the same parameters as those used for the best fit and the equation for two interferences. First the parameters for the low energy dip were used, then the parameters for the high energy dip were used, and finally the two spectra were summed. Again, the sum is not a good fit and the depths are poorly reproduced.

Problems that can arise when attempting to force a fit by using two successive two-state fits are illustrated in Figure 6. Figure 6a shows an enlargement of the spectrum in the region of the dips. In all cases, the experimental spectrum is represented by the dashed line. The lowest trace shows the best two-state fit to the lowest energy dip and the middle trace the best twostate fit to the highest energy dip. The upper trace shows the sum of the two one-state fits. Note that the dips are filled in and the match to the experimental spectrum is poor. If an attempt were made to force the summed spectrum to fit the experimental spectrum, much larger values of the coupling constants would have to be used to counterbalance the filling in.

The parameters that produce a good fit to the experimental spectrum when the correct three-state model is used do not reproduce the spectrum when they are used in two two-state calculations. The parameters listed in Table 2 that produce the excellent fit shown in Figure 5 are tested in the two-state model by using them separately to produce the calculated spectrum shown by the solid line in Figure $6 \mathrm{~b}$. In this calculation, the parameters from Table 2 are used for two calculations with eq 1.3. For the first calculation, the parameters $\gamma_{2}$ and $\epsilon_{\mathrm{F} 2}$ are omitted, leading to a spectrum with only one interference dip caused by state 1 . For the second calculation, the parameters $\gamma_{1}$ and $\epsilon_{\mathrm{F} 1}$ are omitted and the interference dip is caused by state 2 . The two spectra are summed and normalized to the maximum absorbance. Again the dips are washed out, and in addition the bandwidth is poorly reproduced. For spectra containing two interference dips, eqs 1.6 or 1.7 must be used.

\section{Physical and Mathematical Meanings}

The physical meaning and mathematical origins of the interference dips and peaks can be found in three ways. First, the dip can be visualized in terms of interference between wave packets launched on two paths. Second, it can be interpreted in terms of the functional forms of the components of the cross sections. Finally, the two peaks separated by the dip can be explained in terms of a recurrence in the time domain caused by back amplitude transfer from the forbidden surface.

A. Interference between Two Wave Packets. In the simplest terms, the absorption transition to the coupled excited states is reminiscent of the classic double-slit diffraction experiment, where intensity modulations caused by the interference between waves taking paths through different slits are observed. When a wave packet is launched on the allowed state's surface, it can propagate on that surface and transfer amplitude and propagate on the forbidden surface. The interactions between the wave packets on the two surfaces lead to interferences. If the wave packet only propagates on the allowed state (and there was no coupling to the forbidden state), the absorption spectrum would be smooth and featureless. The coupling to the forbidden state gives the electron two primary options on the surfaces in Figure 2: it can stay on the allowed state (route 1), or it can hop from the allowed state to the forbidden state, stay there, and then hop back (route 2). The interference between these two paths (as well as the further paths associated with jumping twice back and forth, etc.) leads to the interference features (dips and peaks in Figures 1, 3, and 4). The interference sign is determined by frequency: at some frequency it is positive, and at others negative.

To elaborate on the mathematical framework, eq 1.3 is expanded as

$$
\begin{aligned}
&-\pi \sigma(\omega)=\operatorname{Im}\left(\frac{\beta}{1-\gamma^{2} \beta \alpha}\right) \approx \\
& \operatorname{Im}(\beta+\beta \gamma \alpha \gamma \beta+\beta \gamma \alpha \gamma \alpha \gamma \beta+\ldots)
\end{aligned}
$$

The terms in this expansion offer a physical interpretation. First, the wave function can fall off directly without even interacting with the forbidden state. There is an amplitude for doing that. Second, it can fall off while interacting with the forbidden state. Specifically, this wave packet falls off on the allowed state, then "jumps" into the forbidden state (through the coupling $\gamma$ ). The wave packet then propagates on the forbidden state (with an amplitude $\alpha$ ) and then finally jumps back to the allowed state, yielding another $\gamma$ factor. The process continues to higher orders, but this is sufficient for our purposes. The key is that the wave packet launched on the allowed state undergoes interference between the term propagated purely on the allowed state and the term that is also propagated on the forbidden state. The two terms are $b+\beta \gamma \alpha \gamma \beta$, so that there will be destructive interference if $\operatorname{Re} \beta \alpha$ is negative.

A less formal approach is to look directly at $C(t)$ and interpret the constructive and destructive interference based on that. $C(t)$ can be written

$$
\begin{array}{r}
C(t)=C_{0}(t)+\int_{0}^{\infty} \int_{0}^{t^{\prime}} \int_{0}^{t^{\prime \prime}} \int_{0}^{t^{\prime \prime \prime}}\left\langle\psi_{0}\right| \exp \left(-i H_{0}\left(t-t^{\prime}-t^{\prime \prime}-\right.\right. \\
\left.t^{\prime \prime \prime}-t^{\prime \prime \prime \prime}\right) \exp \left(-i H_{c} t^{\prime}\right) \exp \left(-i H_{c} t^{\prime \prime}\right) \exp \left(-i H_{c} t^{\prime \prime \prime}\right) \\
\exp \left(-i H_{c} t^{\prime \prime \prime \prime}\right)\left|\psi_{0}\right\rangle \mathrm{d} t^{\prime} \mathrm{d} t^{\prime \prime} \mathrm{d} t^{\prime \prime \prime} \mathrm{d} t^{\prime \prime \prime \prime}+O\left(\gamma^{6}\right)
\end{array}
$$

As before, this (the second term) is interpreted as follows: the first part $\left(\exp \left(-i H_{0} t^{\prime \prime \prime \prime}\right)\right)$ of the second term is associated with propagation of $\Psi_{0}$ on $\mathrm{H}_{0}$, i.e., the allowed state. Then the $t^{\prime \prime \prime}$ 


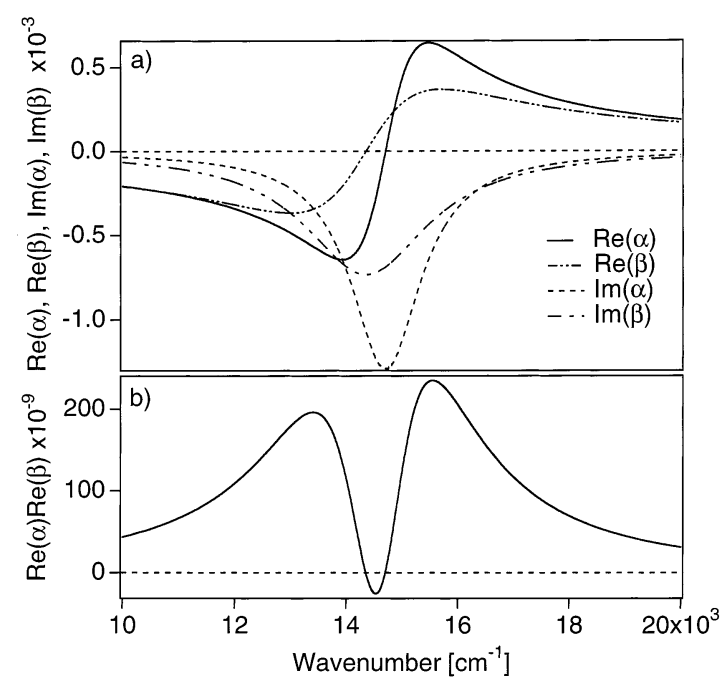

Figure 7. (a) Plots of the real and imaginary parts of $\alpha$ and $\beta$ from the analytical equation (eq 1.3). The interference dip and the double peak result from the product of the real parts of the Lorentzians that change sign in different parts of the spectrum. (b) The product of $\operatorname{Re}(\alpha) \operatorname{Re}(\beta)$ is shown in the bottom panel.

term brings it to the forbidden state, the $t^{\prime \prime}$ term propagates it there, and the $t^{\prime}$ term brings it back to the allowed state, where, depending on the phase it accumulated, it can interfere positively or negatively with the initial (purely allowed state) term, to bring a peak (at some time) or a valley at another time.

B. Components of the Cross Sections. The mathematical reason for the interference dip separated by two peaks is most simply illustrated by examining the analytical formula for the difference spectra (eq 1.3, plotted in Figure 7). When the coupling is small, the difference spectrum becomes

$$
\sigma_{\text {diff }}(\omega) \approx \frac{\gamma^{2}}{\pi} \operatorname{Im}\left(2 \operatorname{Re} \alpha \operatorname{Re} \beta \operatorname{Im} \beta+\operatorname{Im} \alpha \operatorname{Re} \beta^{2}\right)
$$

The real and imaginary parts of $\alpha$ and $\beta$ are plotted in Figure 7a. The dip and peaks originate from the product of $\operatorname{Re} \alpha \operatorname{Re} \beta$ because each component changes sign at a different wavenumber. On each side of the minimum, both $\operatorname{Re} \alpha$ and $\operatorname{Re} \beta$ have the same sign, the product is positive and the constructive interference yields the peaks, as illustrated in Figure $7 \mathrm{~b}$.

It is interesting to note that the dip cannot reach zero absorbance unless $\Gamma=0$. This result can be visualized by following the trend as Re $\alpha$ becomes narrower, the destructive interference occurs over a narrower wavenumber region, and the dip narrows and becomes deeper.

C. Back Amplitude Transfer. The time-domain explanation of the interference dip is based on the short-time autocorrelation function. ${ }^{1}$ When the two surfaces are coupled, the total autocorrelation function decreases more rapidly than it does without coupling (leading to a broader absorption spectrum). Most importantly, it increases to reach a local maximum that is absent in the uncoupled case. The maximum is caused by back population transfer from the forbidden to the allowed diabatic surface. At short times, the wave packet develops appreciable probability on the forbidden diabatic state. The decrease in probability on the allowed surface when the wave packet develops probability on the forbidden surface is responsible in part for the rapid decrease in the magnitude of the autocorrelation function and the broader absorption band. The dip and peaks in the spectrum are related to the changes in the populations of the two surfaces. The population of the allowed state decreases rapidly, reaches a local minimum, and then increases to reach a local maximum at short times (less than $\sim 30 \mathrm{fs}$ ). The initial decrease corresponds to loss of population from the allowed state followed by back transfer from the forbidden state. The autocorrelation function roughly follows the population and also shows a rapid decrease followed by an increase. When transformed to the frequency domain, the rapid decrease results in a broad spectrum, and the small recurrence corresponds to a separation between bands in the frequency domain. ${ }^{26}$ The net result of these effects is to produce two new peaks separated by a dip in the coupled spectrum.

Acknowledgment. This work was made possible by grants from the National Science Foundation (CHE 0206857 to J.I.Z. and CHE 0102623 to J.I.Z. and D.N.) and from the Natural Sciences and Engineering Research Council (to C.R.). We thank Professors F. Wasgestian and W. Assmus for their gift of the doped chromium(III) crystal used in this study.

\section{Appendix A}

In this appendix, the cross section for a molecule containing two undisplaced forbidden states coupled to a displaced allowed state is derived. (The derivation is general, and is also appropriate for the case that there are more than two forbidden states.)

The Hamiltonian is

$$
H=H_{0}+H_{\mathrm{C}}
$$

where

$$
H_{0}=H_{0 \mathrm{~A}}+H_{0 \mathrm{D}}
$$

where $H_{0 \mathrm{~A}}$ is the full many-state Hamiltonian of the allowed state, $H_{0 \mathrm{D}}$ refers to a forbidden electronic state (one in the original derivation, two states now), and $H_{\mathrm{C}}$ is the coupling Hamiltonian.

In addition, it is assumed that the coupling Hamiltonian does not couple directly the two forbidden states $D_{1}$ and $D_{2}$ but instead is associated with a coupling of $\mathrm{D}_{1}$ to the A state and with $\mathrm{D}_{2}$ to the A state. This basically means that the two forbidden states, $\mathrm{D}_{1}$ and $\mathrm{D}_{2}$, are not directly coupled. It is further assumed that the coupling of $\mathrm{D}_{1}$ to $\mathrm{A}$ is a constant, $\gamma_{1}$, and that there is an analogous term for $\mathrm{D}_{2}-\mathrm{A}$ coupling

The equations are derived with as little information as possible on the actual molecular Hamiltonian. It is necessary to find

$$
\left\langle\Psi_{0}|G(E)| \Psi_{0}\right\rangle=\left\langle\Psi_{0}\left|\frac{1}{E-H}\right| \Psi_{0}\right\rangle
$$

where $E$ is complex

$$
E=\omega+i \Gamma
$$

A Born expansion is now used to get

$\frac{1}{E-H}=\frac{1}{E-H_{0}}+\frac{1}{E-H_{0}} H_{\mathrm{C}} \frac{1}{E-H_{0}} H_{\mathrm{C}} \frac{1}{E-H_{0}}+\ldots$

Now the assumption is made that $\mathrm{D}$ includes only two states ( $\mathrm{D}_{1}$ and $\mathrm{D}_{2}$, each with a single vibrational state). Therefore, the Hamiltonian matrix can be written as a $2+N$ matrix, where $N$ is the number of vibrational states used for expanding the acceptor state. The full matrix is then 


$$
\begin{gathered}
H=\left[\begin{array}{cc}
H_{D} & \Delta^{T} \\
\Delta & H_{A}
\end{array}\right]=H_{0}+H_{\mathrm{C}} \\
H_{\mathrm{C}}+U^{\mathrm{T}}+U \\
U=\left[\begin{array}{l}
0 \\
\Delta
\end{array}\right]
\end{gathered}
$$

where $H_{\mathrm{D}}$ is now a $2 \times 2$ diagonal matrix and $\Delta$ is an $N \times 2$ matrix

$$
\Delta_{j I}=\gamma_{I}\left\langle\phi_{D I} \mid v_{j}\right\rangle
$$

and $I=1,2$ is an index over the forbidden state, whereas $j$ is an index over the allowed state's vibrational levels. For the upcoming final part of the derivation, it is important that both forbidden states are undisplaced, i.e., $\phi_{\mathrm{D}}=\phi_{\mathrm{D} 1}=\phi_{\mathrm{D} 2}$, so that $\Delta$ has a very simple form: two identical columns up to a constant. This aspect is discussed later.

$H_{\mathrm{A}}$ is an $N \times N$ diagonal vibrational Hamiltonian. In addition, the initial state has the form

$$
\Psi_{0}=\left[\begin{array}{l}
0 \\
\psi_{0}
\end{array}\right]
$$

where $\psi_{0}$ is the initial state which in coordinate states is simply equal to the ground-state of the vibrational Hamiltonian of the forbidden states. So it follows that

$$
\psi_{0 j}=\left\langle\phi_{D} \mid v_{j}\right\rangle
$$

Thus, up to a constant, the coupling $\Delta$ is equal to the initial wave function

$$
\Delta_{j I}=\psi_{0, j} \gamma_{I}
$$

i.e., in matrix form

$$
\Delta=\psi_{0} \gamma^{T}
$$

(i.e., an $N \times 2$ matrix made as $(N \times 1) \times(1 \times 2)$ matrixes $)$. The final step is to rewrite eq A.5 as

$$
\begin{gathered}
H_{C}=U+U^{T} \\
U=\Psi_{0} \gamma^{T}
\end{gathered}
$$

Accumulating all of these equations, the ingredients to the Green's function are obtained from eq A.4:

$$
\begin{aligned}
& \Psi_{0}{ }^{\mathrm{T}} G \Psi_{0}=\Psi_{0}{ }^{\mathrm{T}} G_{0} \Psi_{0}+\Psi_{0}{ }^{\mathrm{T}} G_{0} H_{C} G_{0} \Psi_{0}+\ldots \\
& \Psi_{0}{ }^{\mathrm{T}} G \Psi_{0}=\Psi_{0}{ }^{\mathrm{T}} G_{0} \Psi_{0}+\Psi_{0}{ }^{\mathrm{T}} G_{0}\left(U+U^{\mathrm{T}}\right) G_{0} \Psi_{0}+ \\
& \Psi_{0}{ }^{\mathrm{T}} G_{0}\left(U+U^{\mathrm{T}}\right) G_{0}\left(U+U^{\mathrm{T}}\right) G_{0} \Psi_{0}+\ldots
\end{aligned}
$$

Note that in each of these terms one of the $U$ can be removed. More explicitly, the initial function $\Psi_{0}$ is in the space of the allowed state (its first two rows, associated with the forbidden states, are empty). $G_{0}$ does not change these, whereas in the coupling term, $U+U^{\mathrm{T}}$, only the term $\left(U^{\mathrm{T}}\right)$ which couples from the allowed state to the forbidden state survives. This implies that all the terms odd in the perturbation vanish, and those that remain have the form

$$
\begin{aligned}
\Psi_{0}{ }^{\mathrm{T}} G \Psi_{0}=\Psi_{0}{ }^{\mathrm{T}} G_{0} \Psi_{0} & +\Psi_{0}{ }^{\mathrm{T}} G_{0} U G_{0} U^{\mathrm{T}} G_{0} \Psi_{0}+ \\
& \Psi_{0}{ }^{\mathrm{T}} G_{0} U G_{0} U^{\mathrm{T}} G_{0} U G_{0} U^{\mathrm{T}} \Psi_{0} \ldots
\end{aligned}
$$

Next the specific form (A.10) is used to give

$$
\begin{aligned}
\Psi_{0}{ }^{\mathrm{T}} G \Psi_{0} & =\Psi_{0}{ }^{\mathrm{T}} G_{0} \Psi_{0}+\Psi_{0}{ }^{\mathrm{T}} G_{0} \Psi_{0} \gamma^{\mathrm{T}} G_{0} \gamma \Psi_{0}{ }^{\mathrm{T}} G_{0} \Psi_{0}+ \\
& \Psi_{0}{ }^{\mathrm{T}} G_{0} \Psi_{0} \gamma^{\mathrm{T}} G_{0} \gamma \Psi_{0}{ }^{\mathrm{T}} G_{0} \Psi_{0} \gamma^{\mathrm{T}} G_{0} \gamma \Psi_{0}{ }^{\mathrm{T}} \Psi_{0} \ldots
\end{aligned}
$$

or finally

$$
\begin{aligned}
\Psi_{0}{ }^{\mathrm{T}} G_{0} \Psi_{0} & =\beta+\beta c \beta+\beta c \beta c \beta+\ldots \\
& =\frac{\beta}{1-c \beta}
\end{aligned}
$$

where $\beta$ has the same meaning as in eq $1.3\left(\Psi_{0}^{\mathrm{T}} G_{0} \Psi_{0}\right)$, whereas " $c$ ", which in the original derivation was simply $\gamma^{2} \alpha$, is here

$$
\gamma^{\mathrm{T}} G_{0} \gamma=\left(\gamma_{1}, \gamma_{2}\right)\left[\begin{array}{ll}
\alpha_{1} & \\
& \alpha_{2}
\end{array}\right]\left[\begin{array}{l}
\gamma_{1} \\
\gamma_{2}
\end{array}\right]=\gamma_{1}^{2} \alpha_{1}+\gamma_{2}^{2} \alpha_{2}
$$

Thus, in conclusion, eq 1.3 is replaced by

$$
\left\langle\Psi_{0}\left|\frac{1}{E-H}\right| \Psi_{0}\right\rangle=\frac{\beta}{1-\left(\gamma_{1}{ }^{2} \alpha_{1}+\gamma_{2}{ }^{2} \alpha_{2}\right) \beta}
$$

or

$$
\sigma(\omega)=-\frac{1}{\pi} \operatorname{Im}\left(\frac{\beta}{1-\left(\gamma_{1}{ }^{2} \alpha_{1}+\gamma_{2}{ }^{2} \alpha_{2}\right) \beta}\right)
$$

\section{Appendix B}

In this appendix, the expression for $\beta$ for a harmonic excitedstate potential surface displaced along several normal coordinates is derived. The derivation starts from the well-known expression

$$
\begin{aligned}
\beta(\omega) \equiv-\left\langle\Psi_{0}\left|\left(H_{0}-\omega-i \Gamma\right)^{-1}\right| \Psi_{0}\right\rangle= \\
\quad-i \int_{0}^{\infty}\left\langle\Psi_{0}\left|\mathrm{e}^{-i\left(H_{0}-\omega\right) t-\Gamma t}\right| \Psi_{0}\right\rangle \mathrm{d} t \\
=-i \int_{0}^{\infty}\left\langle\Psi_{0} \mid \Psi(t)\right\rangle \mathrm{e}^{i \omega t-\Gamma t} \mathrm{~d} t
\end{aligned}
$$

where the wave packet starts at $\psi_{0}$ and is propagated under the zero-order Hamiltonian. The next stage is to notice that on the excited state the motion is uncoupled between the different phonons, so that the overlap function becomes

$$
\left\langle\Psi_{0} \mid \Psi(t)\right\rangle=\prod_{j}\left\langle\eta_{j_{0}} \mid \eta_{j}(t)\right\rangle
$$

where $\eta_{j}$ denotes the individual phonon terms. Because $\eta_{j_{0}}$ is a Gaussian, the overlap is ${ }^{35}$

$$
\left\langle\eta_{j_{0}} \mid \eta_{j}(t)\right\rangle=\exp \left(-\zeta_{j}\left(1-\exp \left(-i \omega_{j} t\right)\right)-\frac{i t \omega_{j}}{2}\right)
$$

thereby completing the proof.

\section{References and Notes}

(1) Reber, C.; Zink, J. I. J. Chem. Phys. 1992, 96, 2681. 5304

(2) Neuhauser, D.; Park, T.-J.; Zink, J. I. Phys. Rev. Lett. 2000, 85

(3) Sturge, M. D.; Guggenheim, H. J.; Pryce, M. H. L. Phys. Rev. B 1970, 2, 2459. 

129.

(4) Bermudez, V. R.; McClure, D. S. J. Phys. Chem. Solids 1979, 40,

(5) Lempicki, A.; Andrews, L.; Nettel, S. J.; McCollum, B. C.; Solomon, E. I. Phys. Rev. Lett. 1980, 44, 1234.

(6) Riesen, H.; Güdel, H. U. Mol. Phys. 1987, 60, 1221.

(7) Meijerink, A.; Blasse, G. Phys. Rev. B 1989, 40, 7288.

(8) Illaramendi, M. A.; Fernández, J.; Balda, R.; Lucas, J.; Adam, J. L. J. Lumin. 1991, 47, 207.

(9) McDonald, R. G.; Stranger, R.; Hitchman, M. A.; Smith, P. W Chem. Phys. 1991, 154, 179.

(10) Illarramendi, M. A.; Balda, R.; Fernández, J. Phys. Rev. B 1993, 47,8411 .

(11) Voda, M.; Garcia-Solé, J.; Vergara, I.; Kaminskii, A.; Mill, B.; Butashin, A. Phys. Rev. B 1994, 49, 3755.

(12) Wexler, D.; Zink, J. I. Inorg. Chem. 1995, 34, 1500.

(13) Hazenkamp, M. F.; Güdel, H. U.; Atanasov, M.; Kesper, U.; Reinen, D. Phys. Rev. B 1996, 53, 2367.

(14) Mendoza, C. R.; Costa, B. J.; Messaddeq, Y.; Zilio, S. C. Phys. Rev. B 1997, 56, 2483.

(15) Stranger, R.; McMahon, K. L.; Gahan, L. R.; Bruce, J. I.; Hambley, T. W. Inorg. Chem. 1997, 36, 3466.

(16) Buñuel, M. A.; Alcalá, R.; Cases, R. Sol. Stat. Commun. 1998, $107,491$.

(17) Kück, S.; Hartung, S.; Hurling, S.; Petermann, K.; Huber, G. Phys. Rev. B 1998, 57, 2203 .
(18) Noginov, M. A.; Loutts, G. B.; Warren, M. J. Opt. Soc. Am. B 1999, $16,475$.

(19) Rodrìguez-Mendoza, U. R.; Rodrìguez, V. D.; Lavin, V.; Martin, I. R.; Nuñez, P. Spectrochim. Acta 1999, A55, 1319.

(20) Gutzov, S.; Wasgestian, F.; Barthel, T.; Assmus, W. Z. Phys. Chem 1998, 205, 41

(21) Schenker, R.; Triest, M.; Reber, C.; Güdel, H. U. Inorg. Chem. 2001, 40, 5787.

(22) Bussière, G.; Reber, C. J. Am. Chem. Soc. 1998, 120, 6306.

(23) Fano, U. Phys. Rev. 1961, 124, 1866.

(24) Fano, U.; Cooper, J. W. Phys. Rev. 1965, 137, A1364

(25) Reber, C.; Zink, J. I. Comments Inorg. Chem. 1992, 13, 177

(26) Zink, J. I.; Shin, K.-S. K. Adv. Photochem. 1991, 16, 119.

(27) Reber, C.; Zink, J. I. J. Phys. Chem. 1992, 96, 571.

(28) Wexler, D.; Zink, J. I.; Reber, C. J. Phys. Chem. 1992, 96, 8757

(29) Jørgensen, C. K. Acta Chem. Scand. 1955, 9, 405.

(30) Jørgensen, C. K. Acta Chem. Scand. 1955, 9, 1362.

(31) Liehr, A. D.; Ballhausen, C. J. Ann. Phys. 1959, 2, 134.

(32) Pryce, M. H. L.; Agnetta, G.; Garofano, T.; Palma-Vittorelli, M. B.; Palma, M. U. Philos. Mag. 1964, 10, 77.

(33) Solomon, E. I.; Ballhausen, C. J. Mol. Phys. 1975, 29, 279.

(34) Illaramendi, M. A.; Fernández, J.; Balda, R. J. Phys.: Cond. Matter 2002, 14, 555

(35) Myers, A. B.; Mathies, R. A.; Tannor, D. J.; Heller, E. J. J. Chem. Phys. 1982, 77, 3857. 